\title{
Scientometrics of drug discovery efforts: pain-related molecular targets
}

\author{
This article was published in the following Dove Press journal: \\ Drug Design, Development and Therapy \\ I July 2015 \\ Number of times this article has been viewed
}

\section{Igor Kissin}

Department of Anesthesiology, Perioperative and Pain Medicine, Brigham and Women's Hospital, Harvard Medical School, Boston, MA, USA
Correspondence: Igor Kissin

Department of Anesthesiology, Brigham and Women's Hospital, 75 Francis Street, Boston, MA 02115, USA

$\mathrm{Tel}+\mathrm{I} 6177325052$

Fax + I 6177340682

Email kissin@zeus.bwh.harvard.edu
Abstract: The aim of this study was to make a scientometric assessment of drug discovery efforts centered on pain-related molecular targets. The following scientometric indices were used: the popularity index, representing the share of articles (or patents) on a specific topic among all articles (or patents) on pain over the same 5-year period; the index of change, representing the change in the number of articles (or patents) on a topic from one 5-year period to the next; the index of expectations, representing the ratio of the number of all types of articles on a topic in the top 20 journals relative to the number of articles in all $(>5,000)$ biomedical journals covered by PubMed over a 5-year period; the total number of articles representing Phase I-III trials of investigational drugs over a 5-year period; and the trial balance index, a ratio of Phase I-II publications to Phase III publications. Articles (PubMed database) and patents (US Patent and Trademark Office database) on 17 topics related to pain mechanisms were assessed during six 5-year periods from 1984 to 2013. During the most recent 5-year period (2009-2013), seven of 17 topics have demonstrated high research activity (purinergic receptors, serotonin, transient receptor potential channels, cytokines, gamma aminobutyric acid, glutamate, and protein kinases). However, even with these seven topics, the index of expectations decreased or did not change compared with the 2004-2008 period. In addition, publications representing Phase I-III trials of investigational drugs (2009-2013) did not indicate great enthusiasm on the part of the pharmaceutical industry regarding drugs specifically designed for treatment of pain. A promising development related to the new tool of molecular targeting, ie, monoclonal antibodies, for pain treatment has not yet resulted in real success. This approach has not yet demonstrated clinical effectiveness (at least with nerve growth factor) much beyond conventional analgesics, when its potential cost is more than an order of magnitude higher than that of conventional treatments. This scientometric assessment demonstrated a lack of real breakthrough developments.

Keywords: analgesics, bibliometrics, biomedical journals, drug design, patents, pharmaceutical industry

\section{Introduction}

Scientometrics analyzes the quantitative aspects of generation, propagation, and utilization of scientific information in order to contribute to a better understanding of the mechanism of research activities; it includes the measurement of scientific output (publications) as well as of the impact of scientific findings on subsequent developments in related areas of research. Scientometric assessments of drugs have been reported previously in a number of publications. ${ }^{1-4}$ Based on such assessments, several scientometric indices have been suggested to demonstrate progress in pharmacotherapy. ${ }^{5-7}$

Over the past century, many new drugs have been introduced for the relief and prevention of pain. However, there is currently the feeling that success in the development of new analgesic drugs has been quite limited despite improvements in our 
understanding of pain mechanisms. ${ }^{8}$ The achievements in drug discovery based on targeting of pain mechanisms have been presented in a number of excellent reviews. ${ }^{9-12}$ The aim of the current study was to assess drug discovery efforts centered on pain molecular targets using specific scientometric indices, developments over the past 30 years were analyzed.

\section{Methods}

Pain mechanisms and their molecular drivers discovered in recent searches for new analgesics were used to collect the appropriate pain targets. They were selected from previous reviews on molecular targets of pain $^{8-10,13}$ and included 17 topics (Table 1). A number of the new drugs belong to old pharmacological groups of analgesics, eg, opioids, nonsteroidal anti-inflammatory drugs, anticonvulsants, and antidepressants. With several exceptions, at best they represent only incremental improvements on old mechanisms. ${ }^{8,9}$ Therefore, most molecular targets related to new drugs from these four groups were not included in the topics of this assessment. However, when development in an old area resulted in discovery of a putative new molecular target, that target was included in the related searches.

The intensity of efforts associated with assessment of pain-related molecular targets was measured in two areas: article publication in biomedical journals as reflected by the PubMed database and patenting as reflected by the US Patent and Trademark Office database. The following scientometric parameters $^{4-7}$ were used.

\section{Popularity index}

The article-related popularity index (PI) is the percentage of articles on a specific topic (pertinent to a pain modulator and its molecular targets) among all articles on pain published over the same 5-year period. Similarly, the patent-related PI is the percentage of patents on a topic among all US patents pertinent to pain.

\section{Index of change}

The index of change (IC) is the percentage change in the number of articles (or patents) on a specific topic during one 5-year period compared with the previous 5-year period.

\section{Index of expectations}

The index of expectations (IE), or Top Journal Selectivity Index, is the ratio of the number of all types of articles on a particular topic in the top 20 journals relative to the number of articles in all $(>5,000)$ biomedical journals on the same topic covered by PubMed over 5 years. It reflects the level of interest in the top journals. An index value $\geq 10$ was selected to represent a high expectation of success. The top 20 journals were selected based on two factors: their rank sorted by impact factor (as indicated by journal citation report for 2013) and the journal specialty area. They included ten journals related to pharmacology and drug discovery, as well as general biomedical and medical journals (also ten journals), as follows: Anesthesiology, Annals of Internal Medicine, Annals of Neurology, Clinical Pharmacology and Therapeutics, Drug Discovery Today, Expert Opinion

Table I Keywords used for searches related to pain modulators and their molecular targets

\begin{tabular}{|c|c|c|}
\hline Number & Topic & Keywords" used in addition to "pain" or "pain and migraine" \\
\hline $\mathrm{I}$ & Purinergic receptors & Purinergic receptors, adenosine receptors, AI, A2, A3, P2Y, P2X \\
\hline 2 & Serotonin ${ }^{\mathrm{b}}$ & Serotonin \\
\hline 3 & Bradykinin & Bradykinin \\
\hline 4 & Substance $P$ & Substance P, NK-I \\
\hline 5 & TRP channels & Transient receptor potential channels, TRPVI, TRPAI, capsaicin \\
\hline 6 & Calcium channels & Calcium channels, T-type, N-type, CaV2.2, CaV3.2 \\
\hline 7 & Cholecystokinin & Cholecystokinin \\
\hline 8 & Cytokines & Cytokines, chemokine, TNF- $\alpha$, IL-I $\beta$ \\
\hline 9 & $\mathrm{GABA}^{\mathrm{b}}$ & GABA, gabapentin, pregabalin \\
\hline 10 & Glutamate ${ }^{\mathrm{b}}$ & Excitatory amino acid agonists, glutamate, AMPA, NMDA, kainate \\
\hline 11 & Botulinum toxins ${ }^{\mathrm{b}}$ & Botulinum toxins, Botox \\
\hline 12 & Cannabinoids & Cannabinoids, CBI, CB2 \\
\hline 13 & CGRPb & Calcitonin gene-related peptide, CGRP, RAMPI \\
\hline 14 & Neurotrophins & Nerve growth factors, BDNF, GDNF, TrkA \\
\hline 15 & Nitric oxide synthase ${ }^{b}$ & Nitric oxide synthase, iNOS \\
\hline 16 & Protein kinases & Protein kinases, PKA, PKC, MAPK, ERK \\
\hline 17 & VGSC & Voltage-gated sodium channels, NAVI.3, NAVI.7, NAVI.8 \\
\hline
\end{tabular}

Notes: a keyword was included if its addition increased the total number of articles by $\geq 2 \%$; "topic was searched with "migraine" added to "pain" term. As a result, the popularity index of a topic marked by "b" can be slightly lower compared with popularity of a topic not marked by "b", despite the similar number of articles for these two topics. Abbreviations: TRP, transient receptor potential; GABA, gamma aminobutyric acid; CGRP, calcitonin gene-related peptide; VGSC, voltage-gated sodium channels. 
on Therapeutic Targets, JAMA: Journal of the American Medical Association, Journal of Clinical Investigation, Journal of Medicinal Chemistry, Journal of Pharmacology and Experimental Therapeutics, Lancet, Nature, Nature Reviews Drug Discovery, New England Journal of Medicine, Neuropharmacology, Pain, Science, Pharmacological Reviews, and Trends in Pharmacological Sciences.

\section{Trial balance index}

The trial balance index (TBI) reflects the balance between the numbers of articles representing different phases of clinical trials for new investigational drugs, ie, the ratio of the number of articles reporting Phase I plus Phase II trials on a topic to the number of articles reporting the Phase III trials on the same topic. Clinical trials of a new investigational drug begin with Phases I and II, and if the results are promising, then the assessment proceeds to Phase III, in which safety and efficacy are studied in a large sample of selected patients. Usually several compounds offered by different companies but acting on the same molecular target undergo clinical trials during the same time interval. In the beginning, articles representing trials of new investigational drugs are limited to Phases I and II; later articles on Phase III trials began to appear and their numbers increase rapidly. The research efforts of the pharmaceutical industry related to a new molecular target are reflected by the total number of new Phase I-III clinical trials. The balance between phases of trials (specifically TBI) indicates whether interest in the development of a target is at its beginning or end. In 2009-2013, the TBI for clinical trials of all investigational drugs covered by PubMed was 2.8. The newer the molecular target at the center of industry interest, the higher the ratio, and vice versa.

\section{Searches}

The above indices related to published articles were calculated on the basis of the results obtained via searches of the PubMed database, ie, the National Library of Medicine's PubMed website (http://www.ncbi.nlm.nih.gov/pubmed). The names of various molecular targets in combination with the keywords "pain" or "pain or migraine" were entered into the search box. For example, for the topic "purinergic receptors" (Table 1), the following keywords were used: "(purinergic receptors OR adenosine receptors OR A1 OR A2 OR A3 OR P2Y OR P2X) AND pain”. If possible, keywords indicated by the National Library of Medicine as "MeSH terms" were used. As a rule, a keyword was included if its addition increased the total number of articles on a topic by $\geq 2 \%$ (above the number already present for this topic due to the previously included keywords). Articles during certain time intervals were counted with the use of the custom range for publication dates and the filter for languages (English). As a rule, all type of articles were considered, with two exceptions: to calculate the Top Journal Selectivity Index, in addition to all types of articles, the articles published in the top 20 journals were also determined; to calculate the TBI, article types were customized to select only those reflecting clinical trial, Phase I, II or III. To identify articles reporting Phase I-III clinical trials of new investigational drugs related to pain, the following two specific approaches were used: (a) in addition to the name of a target, the names of most common disorders in which pain is a predominant symptom were placed into the search box (such as "chronic back pain" OR "chronic muscular-skeletal pain" OR "fibromyalgia" OR "myofascial pain" OR "postherpetic neuralgia" OR "trigeminal neuralgia” OR "diabetic neuropathy" OR “complex regional pain syndrome" OR "central pain"); (b) the PubMed database was searched for so-called "topic-in-title articles", ${ }^{14}$ the titles of which prominently feature pain (such as pain [Title] OR migraine [Title] OR neuralgia [Title]). This was done when there was a need to separate studies in which pain was the primary aim of the trial from studies in which pain was not a primary aim, but pain-related results were reported (for example, studies on an investigational anticancer drug with results related to pain). The articles identified using these two electronic search approaches were inspected manually to determine whether pain was the primary aim.

In addition to publications in biomedical journals, the intensity of efforts associated with the development of painrelated molecular targets was also assessed using the number of related patents in the US Patent and Trademark Office database (http://partft1.uspto.gov/netahtml/PTO/search-adv.htlm). The database was searched using the same keywords used for searching published articles in biomedical journals; the abstract field in the patent database was used for this aim. The number of patents during the 5-year periods (as with journal articles) was determined. To determine the patent-related popularity index, the number of patents on one of the specific pain-related molecular targets (Table 1) over a 5-year period was presented as a percentage of all pain-related patents (independent of specific molecular targets) during the same time interval.

\section{Results}

Table 2 presents the total number of articles and patents on each of the studied topics for the past 30 years (1984-2013), including data on the last two 5-year periods (2004-2008 and 2009-2013). It shows that the total number of articles 





on cytokines $(7,186)$ is the highest, followed by serotonin $(6,241)$, glutamate $(4,489)$, and gamma aminobutyric acid (GABA, 4,263). Two of these four groups also have the highest number of patents, ie, serotonin (135) and glutamate (130). Table 2 also shows that most drugs approved by the US Food and Drug Administration for pain treatment involve serotonin (nine); GABA-related drugs (four) are the next highest. Among the other 15 topics, four have drugs approved for the treatment of pain, but only one drug per topic.

Table 3 presents the article-related IC, demonstrating that over recent 5-year periods (especially 2009-2013), only four of 17 topics showed growth in the number of articles beyond the growth of all PubMed pain-related articles. These topics include purinergic receptors, cytokines, protein kinases, and voltage-gated sodium channels. Only two of these four topics (purinergic receptors and voltage-gated sodium channels) did not exhibit recent rapid growth in publications related to monoclonal antibodies.

When very long periods of time are considered, changes in growth can be better reflected by the PI than by the IC, because the PI takes into account simultaneous changes in pain-related publications as a whole. The article-related PI is presented in Table 4. It demonstrates that in only six of 17 topics did the PI reach $\geq 1.0$ over at least one of the six 5 -year periods. The index maximum was 2.4 for cytokines (2009-2013), 2.0 for serotonin (1999-2003), 1.5 for glutamate (2004-2008), 1.3 for GABA (2004-2008), 1.2 for transient receptor potential
(TRP) channels (2004-2008), and 1.1 for protein kinases (2009-2013). More importantly, in 2009-2013 compared with 2004-2008, the PI for most topics decreased (or at least did not change), with several exceptions: the increases from 2.0 to 2.4 with cytokines, from 0.9 to 1.1 with protein kinases, and from 0.8 to 1.0 with purinergic receptors; in two groups, calcitonin gene-related peptide (CGRP) and neurotrophins, the increases were from 0.4 to 0.5 .

Table 5 presents the IE, demonstrating a feature common to all topics, ie, a gradual decline in expectations. In the three topics with the highest initial IE, this decline was the most profound: TRP channels, from 25.0 (1994-1998) to 12.0 (2009-2013); glutamate, from 23.3 (1994-1998) to 11.4 (2009-2013); and calcium channels, from 19.3 (1994-1998) to 12.0 (2009-2013). In 2009-2013, seven topics have an IE above 10.0, ie, cannabinoids (13.5), bradykinin (13.0), voltage-gated sodium channels (12.3), TRP channels (12.0), calcium channels (12.0), glutamate (11.4), and cholecystoki$\operatorname{nin}$ (11.3). The most peculiar finding for IE is related to the topics with impressive growth in publications on monoclonal antibody-related new investigational drugs, cytokines, and protein kinases; in 2009-2013, the IE for those two topics declined to rather low levels 4.5 (!) and 8.4, respectively.

The efforts of the pharmaceutical industry associated with initial assessment of pain-related investigational drugs are presented in Table 6 the number of articles on Phase I-II and Phase III trials published 2009-2013. The results demonstrate

Table 3 Article-related index of change for topics on pain modulators and their molecular targets

\begin{tabular}{|c|c|c|c|c|c|c|c|}
\hline \multirow[t]{2}{*}{ Number } & \multirow[t]{2}{*}{ Topic } & \multicolumn{6}{|c|}{ Index of change } \\
\hline & & $1984-1988$ & $1989-1993$ & $1994-1998$ & $1999-2003$ & 2004-2008 & 2009-2013 \\
\hline I & Purinergic receptors & $>100^{a}$ & 48 & 95 & 86 & 61 & $>100$ \\
\hline 2 & Serotonin & 36 & 67 & 65 & 83 & 10 & -3 \\
\hline 3 & Bradykinin & 12 & 92 & 13 & 54 & 16 & -17 \\
\hline 4 & Substance P & 77 & 59 & 21 & 28 & 13 & -6 \\
\hline 5 & TRP channels & 46 & $>100$ & 78 & $>100$ & 96 & 45 \\
\hline 6 & Calcium channels & - & $>100$ & $>100$ & $>100$ & $>100$ & 30 \\
\hline 7 & Cholecystokinin & $>100$ & 15 & 32 & 33 & -24 & -27 \\
\hline 8 & Cytokines & $>100$ & $>100$ & $>100$ & $>100$ & 79 & 65 \\
\hline 9 & GABA & 87 & 43 & $>100$ & $>100$ & 91 & 46 \\
\hline 10 & Glutamate & $>100$ & $>100$ & $>100$ & 84 & 62 & 1 \\
\hline II & Botulinum toxins & - & $>100$ & $>100$ & $>100$ & 93 & 23 \\
\hline 12 & Cannabinoids & - & $>100$ & $>100$ & $>100$ & $>100$ & 17 \\
\hline 13 & CGRP & - & $>100$ & 63 & 81 & 74 & 44 \\
\hline 14 & Neurotrophins & - & - & $>100$ & $>100$ & 39 & 58 \\
\hline 15 & Nitric oxide synthase & - & - & $>100$ & 82 & 32 & 40 \\
\hline 16 & Protein kinases & - & $>100$ & $>100$ & $>100$ & $>100$ & 75 \\
\hline 17 & VGSC & - & - & - & $>100$ & $>100$ & 58 \\
\hline
\end{tabular}

Notes: Article-related index of change is the percentage change in number of published articles on a specific topic during a 5-year period compared with the previous similar period; $>100$, indicates more than $100 \%$.

Abbreviations: TRP, transient receptor potential; GABA, gamma aminobutyric acid; CGRP, calcitonin gene-related peptide; VGSC, voltage-gated sodium channels. 
Table 4 Article-related index of popularity for topics on pain modulators and their molecular targets

\begin{tabular}{|c|c|c|c|c|c|c|c|}
\hline \multirow[t]{2}{*}{ Number } & \multirow[t]{2}{*}{ Topic } & \multicolumn{6}{|c|}{ Index of popularity } \\
\hline & & $1984-1988$ & $1989-1993$ & $1994-1998$ & $1999-2003$ & 2004-2008 & 2009-2013 \\
\hline I & Purinergic receptors & 0.3 & 0.3 & 0.4 & 0.5 & 0.6 & 0.8 \\
\hline 2 & Serotonin & I.I & 1.4 & 1.6 & 2.0 & 1.6 & 1.0 \\
\hline 3 & Bradykinin & 0.2 & 0.4 & 0.3 & 0.3 & 0.2 & 0.1 \\
\hline 4 & Substance $P$ & 0.7 & 0.8 & 0.7 & 0.7 & 0.5 & 0.3 \\
\hline 5 & TRP channels & 0.3 & 0.5 & 0.6 & 0.9 & 1.2 & 1.2 \\
\hline 6 & Calcium channels & $<0.1$ & 0.1 & 0.2 & 0.4 & 0.6 & 0.5 \\
\hline 7 & Cholecystokinin & 0.3 & 0.2 & 0.2 & 0.2 & 0.1 & $<0.1$ \\
\hline 8 & Cytokines & 0.3 & 0.7 & 1.2 & 1.7 & 2.0 & 2.4 \\
\hline 9 & GABA & 0.3 & 0.3 & 0.6 & 1.0 & 1.3 & 1.3 \\
\hline 10 & Glutamate & 0.2 & 0.5 & 1.0 & 1.3 & 1.5 & 1.0 \\
\hline 11 & Botulinum toxins & $<0.1$ & $<0.1$ & 0.1 & 0.4 & 0.5 & 0.4 \\
\hline 12 & Cannabinoids & - & - & 0.1 & 0.4 & 0.6 & 0.5 \\
\hline 13 & CGRP & 0.1 & 0.3 & 0.3 & 0.4 & 0.4 & 0.5 \\
\hline 14 & Neurotrophins & - & - & 0.3 & 0.4 & 0.4 & 0.5 \\
\hline 15 & Nitric oxide synthase & - & - & 0.3 & 0.4 & 0.3 & 0.3 \\
\hline 16 & Protein kinases & - & 0.1 & 0.2 & 0.6 & 0.9 & I.I \\
\hline 17 & VGSC & - & - & - & 0.1 & 0.3 & 0.3 \\
\hline
\end{tabular}

Notes: Article-related index of popularity is the percentage of published articles on a specific topic among all articles on pain published over the same 5 -year period. Abbreviations: TRP, transient receptor potential; GABA, gamma aminobutyric acid; CGRP, calcitonin gene-related peptide; VGSC, voltage-gated sodium channels.

a great disproportionality between studies where pain was the primary aim of a trial and studies that presented pain-related results when pain was not the primary aim. The number of articles of the first type is rather low; levels higher than five were reached by only three topics, ie, GABA (17), serotonin (12), and botulinum toxins (11).

Table 7 presents the TBI, indicating whether the efforts of the pharmaceutical industry relating to the development of a new target are at their beginning or already at an end. The table shows that it is possible to calculate the TBI only for two topics, and they are rather low, ie, 0.3 with GABA and 1.4 with serotonin. For comparison, TBI for drugs related to cytokine and protein kinases in general are presented at the bottom of Table 7. This comparison indicates that the discovery efforts of the pharmaceutical industry related to drugs for pain relief are "nearing the end of their road".

Table 5 Index of expectations for topics on pain modulators and their molecular targets

\begin{tabular}{|c|c|c|c|c|c|c|c|}
\hline \multirow[t]{2}{*}{ Number } & \multirow[t]{2}{*}{ Topic } & \multicolumn{6}{|c|}{ Index of expectations } \\
\hline & & $1984-1988$ & $1989-1993$ & $1994-1998$ & $1999-2003$ & 2004-2008 & 2009-2013 \\
\hline I & Purinergic receptors & 6.0 & 9.6 & 10.4 & 15.2 & 11.6 & 6.1 \\
\hline 2 & Serotonin & 12.8 & 14.7 & 6.7 & 7.2 & 5.9 & 6.2 \\
\hline 3 & Bradykinin & 16.9 & 9.6 & 12.8 & 16.1 & 16.7 & 13.0 \\
\hline 4 & Substance $P$ & 11.4 & 15.3 & 14.4 & 14.7 & 8.0 & 9.4 \\
\hline 5 & TRP channels & 19.4 & 22.3 & 25.0 & 22.4 & 16.8 & 12.0 \\
\hline 6 & Calcium channels & - & 14.3 & 19.3 & 16.3 & 13.3 & 12.0 \\
\hline 7 & Cholecystokinin & 9.9 & 9.8 & 13.9 & 10.5 & 5.5 & 11.3 \\
\hline 8 & Cytokines & - & 2.8 & 5.5 & 5.5 & 6.2 & 4.5 \\
\hline 9 & GABA & 14.3 & 13.2 & 14.8 & 17.4 & 10.9 & 8.6 \\
\hline 10 & Glutamate & 15.2 & 23.8 & 23.3 & 18.9 & 12.3 & 11.4 \\
\hline 11 & Botulinum toxins & - & - & - & 5.0 & 4.2 & 2.9 \\
\hline 12 & Cannabinoids & - & - & 18.3 & 18.9 & 16.5 & 13.5 \\
\hline 13 & CGRP & - & - & 7.2 & 12.2 & 11.6 & 9.5 \\
\hline 14 & Neurotrophins & - & - & 13.1 & 13.8 & 8.9 & 8.9 \\
\hline 15 & Nitric oxide synthase & - & - & 18.7 & 11.2 & 9.4 & 5.8 \\
\hline 16 & Protein kinases & - & - & 10.5 & 10.8 & 11.5 & 8.4 \\
\hline 17 & VGSC & - & - & - & 9.3 & 16.5 & 12.3 \\
\hline
\end{tabular}

Note: Index of expectations, ie, the Top Journal Selectivity Index, is the ratio of the number of articles on a particular topic in the top 20 journals relative to the number of articles in all $(>5,000)$ biomedical journals on the same topic covered by PubMed over 5 years.

Abbreviations: TRP, transient receptor potential; GABA, gamma aminobutyric acid; CGRP, calcitonin gene-related peptide; VGSC, voltage-gated sodium channels. 
Table 6 Number of articles on investigational drugs with pain-related results (2009-2013)

\begin{tabular}{|c|c|c|c|c|c|c|c|}
\hline \multirow[t]{2}{*}{ Number } & \multirow[t]{2}{*}{ Topic } & \multicolumn{3}{|c|}{ Pain was primary aim of trial } & \multicolumn{3}{|c|}{$\begin{array}{l}\text { Pain was not primary aim of trial, } \\
\text { but pain-related results were reported }\end{array}$} \\
\hline & & Phase I-II ${ }^{a}$ & Phase III ${ }^{\mathrm{a}}$ & Total & Phase I-II ${ }^{a}$ & Phase III ${ }^{\mathrm{a}}$ & Total \\
\hline $\mathrm{I}$ & Purinergic receptors & 0 & 0 & - & 10 & 6 & 16 \\
\hline 2 & Serotonin & 7 & 5 & 12 & 6 & 3 & 9 \\
\hline 3 & Bradykinin & 0 & 0 & - & 0 & 0 & - \\
\hline 4 & Substance $P$ & 0 & 0 & - & 2 & 0 & 2 \\
\hline 5 & TRP channels & 3 & 1 & 4 & 0 & 0 & - \\
\hline 6 & Calcium channels & 0 & 0 & - & I & I & 2 \\
\hline 7 & Cholecystokinin & 0 & 0 & - & I & 0 & I \\
\hline 8 & Cytokines & I & I & 2 & 60 & 16 & 76 \\
\hline 9 & GABA & 4 & 13 & 17 & I & I & 2 \\
\hline 10 & Glutamate & 0 & 0 & - & 5 & 3 & 8 \\
\hline 11 & Botulinum toxins & 2 & 9 & 11 & I & 2 & 3 \\
\hline 12 & Cannabinoids & I & 0 & 1 & I & I & 2 \\
\hline 13 & CGRP & 3 & 2 & 5 & 0 & 0 & - \\
\hline 14 & Neurotrophins & I & 0 & I & I & 0 & I \\
\hline 15 & Nitric oxide synthase & 0 & 0 & - & 0 & $\mathrm{I}$ & I \\
\hline 16 & Protein kinases & 0 & 3 & 3 & 60 & 9 & 69 \\
\hline 17 & VGSC & 0 & 0 & - & 0 & 0 & - \\
\hline
\end{tabular}

Note: aPhases of clinical trials required for marketing of new drugs.

Abbreviations: TRP, transient receptor potential; GABA, gamma aminobutyric acid; CGRP, calcitonin gene-related peptide; VGSC, voltage-gated sodium channels.

The patent-related IP is presented in Table 8. Four of 17 topics at one of the six 5-year periods had an IP $\geq 2.0$ : serotonin, 3.6 (1994-1998), glutamate, 3.4 (1999-2003), CGRP, 3.3 (2004-2008), and calcium channels, 2.0 (2004-2008). IP values for all of these four topics went down in 2009-2013. As indicated in Table 2, which presents scientometric data on 17 molecular topics in general, the number of pain-related patents is approximately two orders of magnitude lower than that for pain-related article publications. This relationship is mirrored by the total number of articles and total number of patents. For example, the total number of pain-related articles covered by PubMed in 2009-2013 was 144,048 and the total number of pain-related patents covered by the US Patent and Trademark Office was 1,149. When the number of patents on a specific topic is presented as the percentage of patents among all other patents on pain obtained over the same 5-year period (patent-related IP), the values of the patent-related IP and the article-related IP are much closer to each other than the total number of patents and articles. For example, in 2009-2013, the serotonin patent-related IP was 0.9 and the article-related IP was 1.0; with TRP channels, the patent-related IP was 1.2 and the article-related IP was also 1.2; and with glutamate the patent-related IP was 1.6 and the article-related IP was 1.0. An additional observation derived from comparisons of changes in patents and articles is that changes in the patent-related IP on some topics can occur faster than for the article-related IP on the same topic. Several such cases are presented in Figure 1A and B.

\section{Discussion}

Previously we have used a number of scientometric parameters to evaluate changes in the status of various drugs and their methods of administration. ${ }^{5-7}$ This is the first study that has used specific scientometric indices to assess research efforts related to the process of drug discovery; this type of assessment was applied to molecular

Table 7 Balance between Phase I-II trials and Phase III trials in the area of investigational drugs for pain relief (2009-20I3)

\begin{tabular}{|c|c|c|c|c|}
\hline & \multirow[t]{2}{*}{ Area } & \multicolumn{2}{|c|}{ Number of articles } & \multirow[t]{2}{*}{$\overline{T B I^{a}(I-I I / I I I ~ r a t i o)}$} \\
\hline & & Phase I-II & Phase III & \\
\hline \multirow[t]{2}{*}{ Drugs designed for pain relief } & GABA & $4^{b}$ & $13^{b}$ & 0.3 \\
\hline & Serotonin & $7^{\mathrm{b}}$ & $5^{b}$ & 1.4 \\
\hline \multirow[t]{2}{*}{ Some other types of drugs ${ }^{c}$} & Cytokines & 1,070 & 303 & 3.5 \\
\hline & Protein kinases & 1,233 & 256 & 4.8 \\
\hline
\end{tabular}

Notes: ${ }^{\mathrm{a}} \mathrm{TBI}$ is the ratio of the number of articles representing the Phase I plus the Phase Il trials of investigational drugs on a topic to the number of articles representing the Phase III trials on the same topic; bonly articles when pain was a primary aim of a trial; 'not necessarily related to pain.

Abbreviations: GABA, gamma aminobutyric acid; TBI, trial balance index. 
Table 8 Patent-related index of popularity for topics on pain modulators and their molecular targets

\begin{tabular}{|c|c|c|c|c|c|c|c|}
\hline \multirow[t]{2}{*}{ Number } & \multirow[t]{2}{*}{ Topic } & \multicolumn{6}{|c|}{ Index of popularity } \\
\hline & & $1984-1988$ & $1989-1993$ & $1994-1998$ & 1999-2003 & 2004-2008 & 2009-2013 \\
\hline I & Purinergic receptors & - & - & 0.5 & 1.0 & 0.9 & - \\
\hline 2 & Serotonin & 1.4 & 2.8 & 3.6 & 2.2 & 1.3 & 0.9 \\
\hline 3 & Bradykinin & - & 0.7 & 0.5 & 0.8 & 0.6 & 1.0 \\
\hline 4 & Substance $P$ & - & 0.9 & 1.6 & 0.7 & 0.2 & 0.1 \\
\hline 5 & TRP channels & 0.3 & I.I & 1.3 & 0.7 & I.I & 1.2 \\
\hline 6 & Calcium channels & - & 0.5 & 1.8 & I.I & 2.0 & 0.9 \\
\hline 7 & Cholecystokinin & - & 0.5 & 0.1 & - & 0.1 & - \\
\hline 8 & Cytokines & - & - & 0.3 & 1.0 & 0.7 & 0.2 \\
\hline 9 & GABA & - & - & 0.2 & 0.8 & 0.3 & 0.6 \\
\hline 10 & Glutamate & - & - & 2.4 & 3.4 & 1.3 & 1.6 \\
\hline II & Botulinum toxins & - & - & - & 1.7 & 1.3 & 1.0 \\
\hline 12 & Cannabinoids & - & - & - & 0.8 & 1.2 & 1.5 \\
\hline 13 & CGRP & - & - & - & 0.2 & 3.3 & 2.5 \\
\hline 14 & Neurotrophins & - & - & - & 0.1 & 1.0 & I.I \\
\hline 15 & Nitric oxide synthase & - & - & 0.4 & I.I & 0.7 & 0.4 \\
\hline 16 & Protein kinases & - & - & - & 0.6 & 0.3 & 1.0 \\
\hline 17 & VGSC & - & - & - & - & 0.5 & 0.8 \\
\hline
\end{tabular}

Note: Patent-related index of popularity is the percentage of US patents on a specific topic among all other US patents on pain published over the same 5 -year period. Abbreviations: TRP, transient receptor potential; GABA, gamma aminobutyric acid; CGRP, calcitonin gene-related peptide; VGSC, voltage-gated sodium channels.

targets in pain mechanisms. Of 17 topics included in this study, seven demonstrated particularly high activity in the last (2009-2013) of the six 5-year periods. As indicated by Table 2, in 2009-2013 the following topics had more than 1,000 articles: purinergic receptors, serotonin, TRP channels, cytokines, GABA, glutamate, and protein kinases. These seven topics are discussed below.

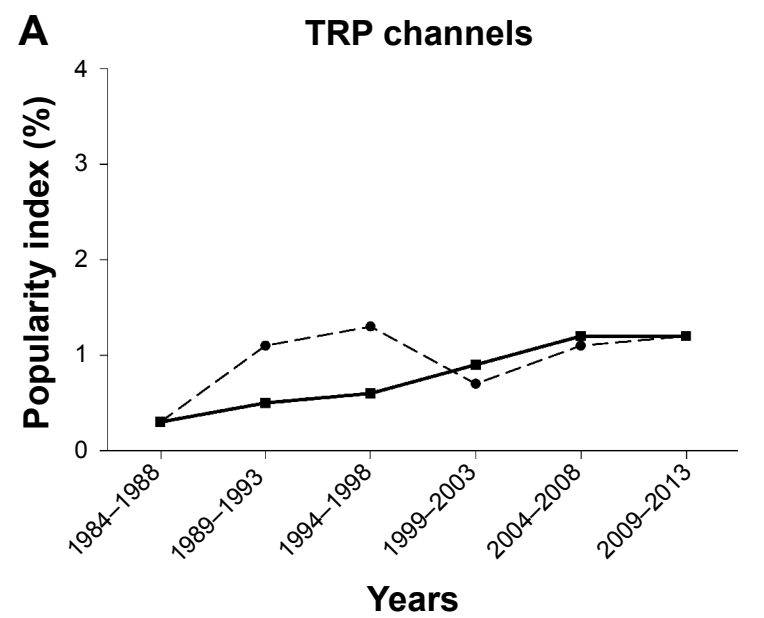

Figure I Time courses of popularity indices related to patents and articles for TRP channels (A) and serotonin (B).

Notes: The article-related popularity index is the percentage of articles on a topic among all articles on pain published over the same 5-year period. Similarly, the patentrelated popularity index is the percentage of patents on a topic among all US patents pertinent to pain. The figure indicates that changes in the patent-related popularity index on these two topics occur faster than for the article-related popularity index.

Abbreviation: TRP, transient receptor potential.

B

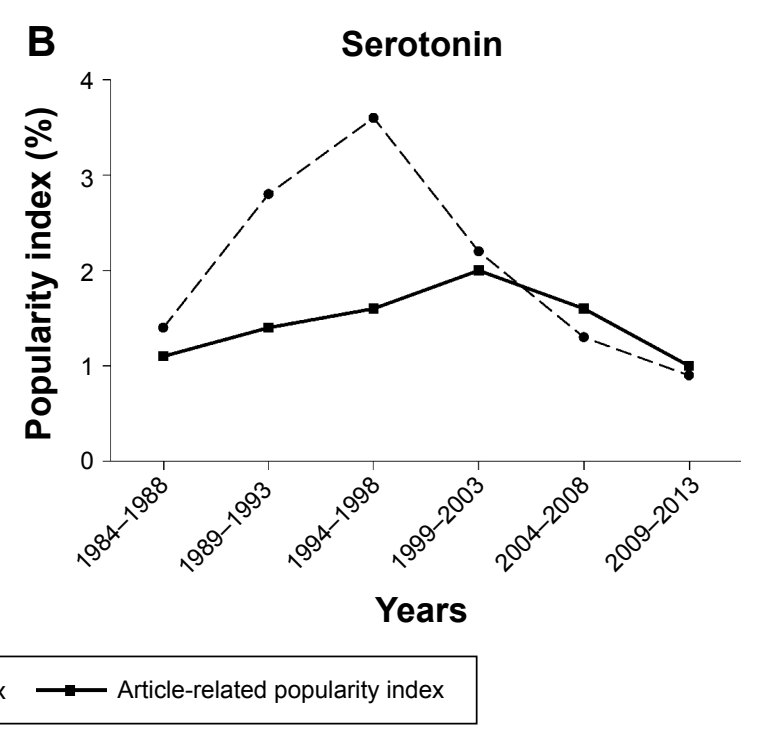

\section{Purinergic receptors}

Over the past 30 years, there has been an impressive growth in pain-related publications associated with these receptors, and both the IC (Table 3) and the PI (Table 4) clearly illustrate this phenomenon. Continuing interest in the role of ATP as a pronociceptive neurotransmitter acting via $\mathrm{P}$ receptors, including roles of these receptors in neuropathic 
pain, was an important factor maintaining this growth. ${ }^{15}$ However, during the most recent 5-year period, there were some important changes indicating a decline. These included a dramatic decrease in the IE to 6.1 in 2009-2013 from 15.2 in 1999-2003 (Table 5). Most importantly, although all 16 articles on Phase I-III studies during 2009-2013 (Table 6) were related to pain, none were focused on drugs designed for pain relief. For example, some of these studies were concerned chiefly with antiplatelet agents.

\section{Serotonin}

This is the area showing the most important achievements over the past 30 years, with nine new drugs approved for the treatment of pain (Table 2). Especially important was the development of sumatriptan and six other follow-on triptans (with a novel selective mechanism of action via the 5-hydroxytryptamine [5-HT] IB/ID receptor), which are very effective in the treatment of acute migraine. The scientometric peak of this development with article-related IC and PI was in 1999-2003 (Tables 3 and 4). With patents the peak was earlier, ie, in 1994-1998 (Table 8). However, after these peaks, the related indices began to decline (Tables 3, 4, and 8). The idea that combined blockade of 5-HT and noradrenaline might be useful in the treatment of pain ${ }^{16}$ led to approval of the use of antidepressants with such properties, such as duloxetine and milnacipran, in several pain syndromes (diabetic neuropathy, fibromyalgia, chronic low back pain, osteoarthritis). Agents acting at various subtypes of the 5-HT receptor or changing 5-HT uptake mechanisms continue to generate interest as potential avenues for the development of new analgesics. In 2009-2013, there were 12 Phase I-III articles on serotonin-related investigational drugs in which pain was the primary aim of the study (Table 6). However, the serotonin TBI of 1.4 is very low. Over the more recent 5-year periods, the serotonin IE was rather low, at 5.9 and 6.2 in 2004-2008 and 2009-2013, respectively (Table 5). The patent-related PI also declined since its peak of 3.6 in 1994-1998; in 2009-2013 it was only 0.9 (Table 8).

\section{TRP channels}

Topical capsaicin has long been used to relieve pain. However, only with an understanding of the mechanism of this effect as the action via one of the types of the TRP ion channels, TRPV1, did these channels become targets for development of new analgesics. The most intensive efforts were directed toward the development of TRPV1 antagonists. Probably as a result of this development, the related IC and IE indices were very high (Tables 3 and 5), especially the IE, which reached 25.0 in 1994-1998. However, many TRPV1 antagonists demonstrate prohibitive side effects, leading to their withdrawal from clinical trials. ${ }^{17}$ As a result, the TRP channel-related high IC levels decreased from $>100$ in 2004-2008 to 45 in 2009-2013 (Table 3); and the IE in the last 5-year period, although still high, also declined to 12.0 (Table 5). The articles on pain-related Phase I-III studies include only four items in 2009-2013 (Table 6). The development of new drugs aimed at the TRPV1 target continued in the area of agonists; ${ }^{18}$ one new medication, the capsaicin $8 \%$ patch, has been approved by the US Food and Drug Administration (Table 2).

\section{Gamma-aminobutyric acid}

Because GABA's principal role is reducing neuronal excitability, it had been the center of many developments aimed at creating new analgesics. There are four drugs in this group specifically approved for the treatment of pain (Table 2). Gabapentin and pregabalin are antiseizure drugs that consist of a modified GABA molecule and are designed to be centrally active GABA agonists. ${ }^{19}$ Similar to many other anticonvulsants (such as carbamazepine) they are effective in the treatment of chronic pain. Valproate, another anticonvulsant that increases cerebral GABA levels, was recently approved for migraine prophylaxis. However, the exact mechanisms underlying the analgesic effects of these antiseizure drugs are not known. For example, gabapentin and pregabalin may work through calcium channels, but they also modulate other analgesic targets, including TRP channels, N-methyl-D-aspartate receptors, protein kinase $\mathrm{C}$, and inflammatory cytokines. ${ }^{20}$ As a general rule related to all 17 topics, with improvement in our knowledge regarding the exact mechanism underlying the analgesic effects of specific drugs, the presented topics might be classified under different titles. During the period 1994-2008, the GABA-related IC and PI indices grew at a rapid rate; however, that was not the case in 2009-2013 (Tables 3 and 4). The index of expectations declined from 17.4 in 1999-2003 to 8.6 in 2009-2013 (Table 5). Although the number of GABA-related articles on Phase I-III studies in 2009-2013 was not extremely high (17 articles), it was the highest among all 17 topics (Table 6). At the same time, the TBI index was very low $(0.3$, Table 7), indicating that influx of Phase I and Phase II studies had already declined profoundly.

\section{Glutamate}

This excitatory neurotransmitter plays an important role in the modulation of pain. The analgesic action of ketamine 
(an N-methyl-D-aspartate receptor antagonist developed as a general anesthetic) has been known for almost 50 years, but its therapeutic effect in postoperative pain was not convincingly confirmed until recently. ${ }^{21}$ This drug has a completely novel mechanism of action, but no follow-on drugs (similar drugs acting on the same molecular target, like triptans) have been approved. The initial high hopes associated with the development of new glutamate-related pain relievers are reflected in high IE values of up to 23.3 in 1994-1998; however, by 2009-2013, this index had declined to 11.4 (Table 5). All other scientometric indices were rather low in 2009-2013: the value of IC was only one. (Table 3), the PI decreased from 1.5 in 2004-2008 to 1.0 (2009-2013) (Table 4). In 2009-2013, there were no articles on Phase I-III studies in which pain was the primary aim of the trial (Table 6).

\section{Cytokines}

Members of this group of small proteins serve as intercellular chemical messengers, acting through specific receptors and mostly produced by a variety of immune cells in response to injury and inflammation. As indicated in Table 2, cytokines show the maximal number of publications among all 17 topics: 3,410 in 2009-2013 and a total of 7,186 (for all 5 -year periods). The rapid growth of cytokine-related publications over the past 30 years is well reflected in the high values of the IC and PI indices (Tables 3 and 4). However, two other indices do not yet indicate very fruitful development: the IE is very low (Table 5) and the number of Phase I-III studies where pain was the primary aim in 2009-2013 was also very low (just two articles), at a time when the number of articles with pain-related results, but not with pain as the primary aim, was very high, at 76 articles (Table 6). These two indices show that at present there are low expectations for drugs designed as cytokine-related pain relievers. The enthusiasm of the pharmaceutical industry is mostly directed toward cytokine-related drugs designed for the treatment of various types of cancers and rheumatoid arthritis; these drugs were not designed as pain-relieving agents.

\section{Protein kinases}

These enzymes change the function of a protein by adding phosphate groups. Many drugs that inhibit specific kinases have been developed for the treatment of cancer and various inflammatory disorders. Some of them are small molecules and others are monoclonal antibodies (biologics). As evidenced by the protein kinase-related IC and PI (Tables 3 and 4), and similar to cytokines, this topic has seen an impressive rise over each 5-year period, although protein kinase-related expectations are not high (IE 8.4 in 2009-2013, Table 5). The number of articles on Phase I-III trials for 2009-2013 in which pain was the primary aim of the study was very low (three articles), at a time when there were 69 pain-related articles on trials in which the primary aim was not pain. This indicates relatively low interest in protein kinase-related approaches to design drugs for the treatment of pain.

\section{Other topics}

For topics with less than $<1,000$ articles in 2009-2013, several points should be discussed. The topic of calcium channels saw an important development, ie, the introduction of ziconotide, an analgesic with a novel mechanism of action of selectively blocking CaV 2.2 calcium channels. However, its utility is very limited because it is used intrathecally and has many serious side effects. The introduction of ziconotide stimulated the search for analgesics among agents blocking calcium channels. As a result, the related PE index for calcium channels, although it had declined since 1994-2003, nevertheless was still high in 2009-2013 at 12.0 (Table 5).

The topic of voltage-gated sodium channels is also active, with high IC and IE indices (Tables 3 and 4). Although the use of cannabis against pain dates back several millennia, in the last few decades research aimed at the treatment of chronic and neuropathic pain has focused on the endocannabinoid system, especially on the activation of $\mathrm{CB}_{2}$ receptor. $\mathrm{CB}_{2}-$ mediated antinociceptive effects seem devoid of any central psychotropic action, thus minimizing the $\mathrm{CB}_{1}$-associated adverse effects. ${ }^{22}$ As indicated in Table 2, the number of articles on cannabinoids (including $\mathrm{CB}_{1}$, and $\mathrm{CB}_{2}$, Table 1) in 2009-2013 reached 651.

The topic of botulinum toxins had a high level of 20092013 articles on Phase I-III trials in which pain was the primary aim, ie, eleven articles (Table 6). This is the result of multiple trials related to the use of botulinum toxin injections for prevention of chronic migraine. ${ }^{23}$ At the same time, the IE level for this topic was exceptionally low, at 2.9 in 2009-2013 (Table 5). CGRP is a potent vasodilator and can function in the transmission of pain. Elevated levels of CGRP have been reported in migraine, and recently developed CGRP receptor antagonists have shown promising results in acute treatment of migraine. ${ }^{24}$ That is the most likely explanation for the exceptionally high patent-related PIs for CGRP in 2004-2008 and in 2009-2013 (Table 8).

Monoclonal antibodies are now a promising and rapidly growing category of targeted therapeutic agents, ${ }^{25}$ mostly for cancer and autoimmune diseases. Three of the 17 topics presented in Table 2 include multiple monoclonal antibodyrelated articles: cytokines, protein kinases, and neurotrophins. Usually, they report pain-related results that are secondary to 
the direct effect of these agents on a cancer or autoimmune disease. Only a limited number of studies used this new tool of targeting to aim at pain mechanisms. One of the most exciting developments in this regard has been targeting the nerve growth factor (NGF) with several monoclonal antibodies, especially to relieve pain associated with osteoarthritis, low back pain, and neuropathic pain. ${ }^{26,27}$ Although these studies provide evidence that inhibition of NGF-mediated processes with monoclonal antibodies is a valid approach to suppressing pain, they also made clear that the development of new analgesics depends on the answers to two critical questions, ie, to what extent is the pain relief clinically meaningful, and whether the use of these types of drugs makes economic sense. It looks like the answers to these questions are not encouraging. Pain relief with the monoclonal antibodies tanezumab or fulranumab in osteoarthritis or neuropathic pain is at 1-2 points on the 11-point scale, ${ }^{26,27}$ while their potential cost is more than an order of magnitude higher than that of conventional pain treatment. This combination of factors is probably the reason for the relatively low levels of expectations for topics related to monoclonal antibodies: neurotrophins, protein kinases, and cytokines (IE 8.9, 8.4, and 5.8, respectively).

The scientometric indices used to identify signs of progress in the therapeutics are based on the link between the number of publications and the progress in pharmacotherapy. However, this link is inherently weak. This weakness is underlined by the fact that the mere number of publications does not differentiate between publications characterizing a drug in a positive or negative way. In addition, many drug trials are never published. Another limitation of the present analysis is that it is based only on two databases, ie, PubMed and the US Patent and Trademark Office.

In conclusion, only once over the past 30 years did the process of drug discovery aimed at pain-related molecular targets achieve a substantial degree of success. Sumatriptan, patented in 1985-1989, demonstrated a novel selective mechanism of action, arising from a better understanding of the mechanism of an existing analgesic $\operatorname{drug}^{8}$ plus clinical acceptability, resulting in US Food and Drug Administration approval of multiple follow-on drugs. This degree of success was not achieved with any other research developments aimed at pain-related molecular targets. The scientometric indices used in this study indicate that the progress in this direction is still very limited. Publications regarding promising developments in the new area of molecular targeting (ie, monoclonal antibodies) have not yet provided a sufficient basis to assess its success in the treatment of pain. This type of targeting has not demonstrated clinical effectiveness well above that of conventional analgesics at a time when the potential cost of such treatment is more than an order of magnitude higher than that of the conventional treatments. Thus, achievements in drug discovery based on targeting of pain mechanisms still demonstrate a lack of real breakthrough developments.

\section{Disclosure}

The author reports no conflicts of interest in this work.

\section{References}

1. Kissin I. Can a bibliometric indicator predict the success of a drug? Scientometrics. 2011;86:785-795.

2. Kissin I, Bradley EL Jr. Top Journals Selectivity Index: is it acceptable for drugs beyond the field of analgesia? Scientometrics. 2011;88: 589-597.

3. Kissin I, Bradley EL Jr. Top Journal Selectivity Index and 'me-too' drugs. Scientometrics. 2012;91:131-142.

4. Kissin I. An early indicator of drug success: Top Journal Selectivity Index. Drug Des Devel Ther. 2013;7:93-98.

5. Correll DJ, Vlassakov KV, Kissin I. No evidence of real progress in treatment of acute pain, 1993-2012: scientometric analysis. J Pain Res. 2014;7:199-210.

6. Kissin I. Scientometric assessment of drugs for chronic pain, 1979-2013; rapid growth of publications, paucity of successful drugs. J Pain Res. 2014; 7:505-514.

7. Vlassakov KV, Kissin I. Scientometrics of anesthetic drugs and their techniques of administration, 1984-2013. Drug Des Devel Ther. 2014;8: 2463-2473.

8. Kissin I. The study on the development of new analgesics over the past 50 years: a lack of real breakthrough drugs. Anesth Analg. 2010:110: 780-789.

9. Hill RG. Analgesic drugs in development. In: McMahon SB, Kolzenburg M, editors. Wall and Melzack's Textbook of Pain. 5th ed. Philadelphia, PA, USA: Elsevier Churchill Livingstone; 2006.

10. Dray A.Pharmacologicalmodulation of pain. In:BasbaumAI,Bushnell MC, editors. Science of Pain. Amsterdam, The Netherlands: Elsevier; 2009.

11. Heinzmann S, McMahon SB. New molecules for the treatment of pain. Curr Opin Support Palliat Care. 2011;5:111-115.

12. Hayes AG, Arendt-Nielsen L, Tate S. Multiple mechanisms have been tested in pain - how can we improve the chances of success? Curr Opin Pharmacol. 2014;14:11-17.

13. Burgess G, Williams D. The discovery and development of analgesics: new mechanisms, new modalities. J Clin Invest. 2010;120: 3753-3759.

14. Kissin I, Gelman S. Chronic postsurgical pain: still a neglected topic? J Pain Res. 2012;5:473-489.

15. Jarvis MF. The neural-glial purinergic receptor ensemble in chronic pain states. Trends Neurosci. 2010;33:48-57.

16. Briley M. New hope in the treatment of painful symptoms in depression. Curr Opin Investig Drugs. 2003;4:42-45.

17. Kaneko Y, Szallasi A. Transient receptor potential (TRP) channels: a clinical perspective. Br J Pharmacol. 2014;171:2474-2507.

18. Kissin I, Szallasi A. Therapeutic targeting of TRPV1 by resiniferatoxin, from preclinical studies to clinical trials. Curr Top Med Chem. 2011;11: 2159-2170.

19. McNamara. Pharmacotherapy of the epilepsies. In: Brunion L, ChabnerBA, Knollmann BC, editors. Goodman and Gilman's Pharmacological Basis of Therapeutics 12th ed. New York, NY, USA: McGraw-Hill; 2011.

20. Kukkar A, Bali A, Singh N, Jaggi AS. Implications and mechanism of action of gabapentin in neuropathic pain. Arch Pharm Res. 2013;36: 237-251.

21. Bell RF, Dahl JB, Moore RA, Kalso E. Perioperative ketamine for acute postoperative pain. Cochrane Database Syst Rev. 2006;1: CD004603. 
22. Luongo L, Maione S, DiMarzo V. Endocannabinoids and neuropathic pain: focus on neuron-glia and endocannabinoid-neurotrophin interactions. Eur J Neurosci. 2014;39:401-408.

23. Cernuda-Morollón E, Ramón C, Larrosa D, Alvarez R, Riesco N, Pascual J. Long-term experience with onabotulinumtoxinA in the treatment of chronic migraine: what happens after one year? Cephalalgia. November 27, 2014. [Epub ahead of print].

24. Edvinsson L. CGRP receptor antagonists and antibodies against CGRP and its receptor in migraine treatment. Br J Clin Pharmacol. March 3, 2015. [Epub ahead of print].

25. Nelson AL, Dhimolea E, Reichert JM. Development trends for human monoclonal antibody therapeutics. Nat Rev Drug Discov. 2010;9: $767-774$.
26. Wang H, Romano G, Frustaci ME, et al. Fulranumab for treatment of diabetic peripheral neuropathic pain: a randomized controlled trial. Neurology. 2014;83:628-637.

27. Spierings EL, Fidelholtz J, Wolfram G, Smith MD, Brown MT, West CR. A phase III placebo- and oxycodone-controlled study of tanezumab in adults with osteoarthritis pain of the hip or knee. Pain. 2013;154: 1603-1612.

28. Iskedjian M, Bereza B, Gordon A, Piwko C, Einarson TR. Meta-analysis of cannabis based treatments for neuropathic and multiple sclerosisrelated pain. Curr Med Res Opin. 2007;23:17-24.

\section{Publish your work in this journal}

Drug Design, Development and Therapy is an international, peerreviewed open-access journal that spans the spectrum of drug design and development through to clinical applications. Clinical outcomes, patient safety, and programs for the development and effective, safe, and sustained use of medicines are a feature of the journal, which has also been accepted for indexing on PubMed Central. The manuscript management system is completely online and includes a very quick and fair peer-review system, which is all easy to use. Visit http://www.dovepress.com/testimonials.php to read real quotes from published authors.

Submit your manuscript here: http://www.dovepress.com/drug-design-development-and-therapy-journal 